\title{
Watch Out for Post-crisis Regulatory Euphoria
}

Dear readers,

although the crisis is not over yet it seems to be giving up as signs of economic upturn have been flashing lately. Logically, analysts and regulatory authorities seem to have shifted the scope of their interest. It is not research for roots of crisis any more - they are now formulating lessons learnt to shape the future of financial system. Similarly to historical periods of economic downturn nowadays we can hear voices calling for sound and more strict regulation. While this is perfectly reasonable it raises a question whether to hold appropriate the chosen trend of fixing the financial system. What are the most important issues?

Last decade of the twentieth century as well as the beginning of the new millennium have both enjoyed important deregulation of financial markets which, in my opinion, not only brought revival to these markets but also had a very positive impact on the whole economy. We may only speculate on what would have happened then if we had set the regulatory rules in the form being considered today. The course of the crisis might have taken a different direction but the shape of financial markets and the whole economy would have been different, too. When contemplating more strict regulation we have to keep in mind the risks of negative impact on financial market and operating investor behaviour while not forgetting about expenses induced by the changes in regulation. Regulation promoters would certainly object that bad regulation induces vast losses and that efficient market benefits more than outweigh costs related to regulation. This objection cannot be put into question and I do not intend to advocate regulation cancellation. The main danger in stake is the risk of regulation over-tightening in the current "post-crisis euphoria".

All regulatory amendments must be thoroughly considered before adoption. They must not be too specific while being very simple, they must look ahead in the future while leaving space for innovations and without over-medication of investor responsibility. The quality of being general and relatively simple at the same time is far from being met with today's regulatory rules, their complexity and sophistication is such that they become sole domain of a very narrow scope of people in regulated and regulatory institutions. The rest of population lacks not only 
knowledge about them but even capacity to understand. The regulation is not easily enforced and disputes often result in long court litigations that overemphasize procedural aspects over true matter. All these aspects amount higher costs of regulation. Additional amendments to existing rules often make the situation even worse.

How can the regulation rules look ahead in the future? Regulatory changes tend to be reactive when fixing a specific problem. We should however notice that both parties learn lessons for the mistake. Regulators and market participants, none of them tend to reproduce the same mistake. If I simplify a bit I reckon that even if we did not change the current regulation there would be no other similar crisis rising from the same roots. And no matter if these roots were conscious or unconscious underestimating of any risk. This is why any regulatory amendment should not only reflect past problems but should with utmost importance predict issues and failures based on the past experience and take them into consideration when formulating new rules. Not forgetting about the prerequisite of general validity, once again. Regulatory rules can only set a certain framework. We may not expect them to make financial markets operate in an efficient way and they may never protect every single investor. Neither can any law abolish crime nor any traffic rule stop speeding. Regulation itself does not make a perfect market. Market participants always have their own stake and make the difference, and therefore it is so important to underline their code of ethics and moral judgements, again and again over time. And we have to keep these connected with responsibility. Over-emphasising regulation may lead to an unexpected drop in vigilance by investors who could misleadingly rely on regulation while assessing risks to be low.

I am aware of the fact that it is by far easier to discuss general principles than formulate regulatory rules. And this is even more true today when rules are to a great extent created at the international level (which is given by the nature of financial markets). Such a situation leaves little space for national legislation. We should however remind regulatory authorities who often remind financial market participants to be precautious to do so when setting regulatory rules.

doc. Ing. Petr Dvořák, Ph.D.

Dean

Faculty of Finance and Accounting, University of Economics, Prague 